\title{
Preoperative Cytologic Diagnosis of Warthin-like Variant of Papillary Thyroid Carcinoma
}

Jisup Kim · Beom Jin Lim Soon Won Hong · Ju Yeon Pyo

Department of Pathology, Gangnam Severance Hospital, Yonsei University College of Medicine, Seoul, Korea

\begin{abstract}
Background: Warthin-like variant of papillary thyroid carcinoma (WLV-PTC) is a relatively rare variant of papillary thyroid carcinoma with favorable prognosis. However, preoperative diagnosis using fine-needle aspiration (FNA) specimens is challenging especially with lymphocytic thyroiditis characterized by Hürthle cells and lymphocytic background. To determine a helpful cytological differential point, we compared WLV-PTC FNA findings with conventional papillary thyroid carcinoma with lymphocytic thyroiditis (PTC-LT) and conventional papillary thyroid carcinoma without lymphocytic thyroiditis (PTC) regarding infiltrating inflammatory cells and their distribution. Preoperative diagnosis or potential for WLV-PTC will be helpful for surgeons to decide the scope of operation. Methods: Of the 8,179 patients treated for papillary thyroid carcinoma between January 2007 and December 2012, 16 patients (0.2\%) were pathologically confirmed as WLV-PTC and four cases were available for cytologic review. For comparison, we randomly selected six PTC-LT cases and five PTC cases during the same period. The number of intratumoral and background lymphocytes, histiocytes, neutrophils, and the presence of giant cells were evaluated and compared using conventional smear and ThinPrep preparations. Results: WLV-PTC showed extensive lymphocytic smear with incorporation of thyroid follicular tumor cell clusters and frequent histiocytes. WLV-PTC was associated with higher intratumoral and background lymphocytes and histiocytes compared with PTC-LT or PTC. The difference was more distinct in liquid-based cytology. Conclusions: The lymphocytic smear pattern and the number of inflammatory cells of WLV-PTC are different from those of PTC-LT or PTC and will be helpful for the differential diagnosis of WLV-PTC in preoperative FNA.
\end{abstract}

Key Words: Warthin-like variant; Thyroid cancer, papillary; Hashimoto disease; Biopsy, fine-needle
Warthin-like variant of papillary thyroid carcinoma (WLVPTC) was first described by Apel et al. ${ }^{1}$ in 1995 and named "warthin-like" due to the presence of abundant lymphocytes interspersed in oncocytic follicular epithelial cells resembling the warthin tumor of salivary glands. It is a relatively rare variant of papillary thyroid carcinoma with only 95 cases published in the English literature. ${ }^{2}$ This tumor expresses the product of the RET/PTC fusion gene, indicating that it is a variant of papillary thyroid carcinoma. ${ }^{3}$ WLV-PTC is a favorable prognostic variant ${ }^{1,3,4}$ although ominous behavior has also been reported. ${ }^{5}$ The diagnosis of WLV-PTC is relatively simple due to its characteristic morphology. However, preoperative diagnosis using fine-needle aspiration (FNA) samples is challenging because abundant lymphocytes and oncocytic follicular epithelial cells (Hürthle cells) are observed in various lesions associated with lymphocytic thyroiditis. In this study, we compared FNA findings of WLVPTC, conventional papillary thyroid carcinoma with lympho- cytic thyroiditis (PTC-LT), and conventional papillary thyroid carcinoma without lymphocytic thyroiditis (PTC). The characteristics of infiltrating inflammatory cells and their distribution were analyzed to evaluate their usefulness for differential diagnosis. Preoperative differential diagnosis of WLV-PTC will be helpful for surgeons to determine the optimal scope of operation.

\section{MATERIALS AND METHODS}

\section{Patients and cases}

We retrieved 8,179 papillary thyroid carcinoma cases from the Thyroid Cancer Center database, Gangnam Severance Hospital, Yonsei University College of Medicine, Seoul, Korea between January 2007 and December 2012. Based on the final pathological diagnosis, 16 patients $(0.2 \%)$ were WLV-PTC including outside consultation cases. We were able to reexamine cytology slides from four of the patients. Six PTC-LT cases and five PTC 
cases were randomly selected for comparison (Table 1). Each case had been prepared for both conventional smear and liquid-based cytology (ThinPrep, Hologic, Bedford, MA, USA). The Institutional Review Board of Gangnam Severance Hospital (local IRB number: 3-2017-0235) approved this retrospective study and informed consent was waived.

\section{Microscopic evaluation}

Three slides (two conventional smears and one ThinPrep) were reviewed in each case. The number of background lymphocytes within tumor clusters as well as the number of histiocytes and neutrophils was counted in 10 high power fields (HPFs, $\times 200)$ in each sample slide. Tumor clusters composed of at least five tumor cells were included in this counting. The presence or absence of background giant cells was recorded. In each case, the average number of inflammatory cells per one HPF was recorded and separated into low and high groups for comparison. The cut-off points are shown in Tables 2 and 3.

Table 1. Baseline clinicopathologic characteristics of each case

\begin{tabular}{|c|c|c|c|c|c|c|}
\hline Case No. & Age $(y r) / S e x$ & Tumor size $(\mathrm{cm})$ & Sonographic feature & FNA diagnosis $^{a}$ & Pathologic diagnosis & Associated thyroiditis \\
\hline 1 & $46 / F$ & 1.0 & Irregular shaped calcified nodule & VI. PTC & PTC-LT & Present \\
\hline 2 & $45 / F$ & 0.9 & Suspicious malignant nodule & VI. PTC & PTC-LT & Present \\
\hline 3 & $46 / F$ & 0.6 & Suspicious malignant nodule & V. Suspicious PTC & PTC-LT & Present \\
\hline 4 & $53 / F$ & 0.4 & Suspicious lesion & VI. PTC & PTC-LT & Present \\
\hline 5 & $53 / F$ & 0.6 & Suspicious nodule & V. Suspicious PTC & PTC-LT & Present \\
\hline 6 & $62 / F$ & 0.4 & Taller than wider hypoechoic nodule & VI. PTC & PTC-LT & Present \\
\hline 7 & $76 / F$ & 0.5 & Suspicious nodule & VI. PTC & PTC & Absent \\
\hline 8 & $44 / F$ & 0.7 & Suspicious malignant nodule & V. Suspicious PTC & PTC & Absent \\
\hline 9 & $49 / F$ & 0.6 & Suspicious lesion & VI. PTC & PTC & Absent \\
\hline 10 & $70 / \mathrm{M}$ & 1.0 & Cancer nodule & VI. PTC & PTC & Absent \\
\hline 11 & $57 / F$ & 0.3 & Suspicious lesion & VI. PTC & PTC & Absent \\
\hline 12 & $33 / F$ & 1.4 & Suspicious nodule & VI. PTC & WLV-PTC & Present \\
\hline 13 & $59 / F$ & 0.3 & Low suspicious nodule & V. Suspicious PTC & WLV-PTC & Absent \\
\hline 14 & $40 / F$ & 0.6 & Oval shaped mass with poor enhancement & V. Suspicious PTC & WLV-PTC & Absent \\
\hline 15 & $48 / F$ & 0.4 & Suspicious nodule & VI. PTC & WLV-PTC & Present \\
\hline
\end{tabular}

FNA, fine-needle aspiration; F, female; PTC, conventional papillary thyroid carcinoma without lymphocytic thyroiditis around the tumor; PTC-LT, conventional papillary thyroid carcinoma with lymphocytic thyroiditis around the tumor; M, male; WLV-PTC, Warthin-like variant of papillary thyroid carcinoma.

aDiagnostic categories according to the Bethesda system for reporting thyroid cytopathology.

Table 2. Comparison of inflammatory cell components among the papillary thyroid cancer groups in conventional smears

\begin{tabular}{|c|c|c|c|c|c|}
\hline Inflammatory cell component & WLV-PTC $(n=4)$ & PTC-LT (n=6) & PTC $(n=5)$ & $\begin{array}{l}\text { WLV-PTC vs PTC-LT } \\
\text { p-value }\end{array}$ & $\begin{array}{c}\text { WLV-PTC vs PTC } \\
\text { p-value }\end{array}$ \\
\hline \multicolumn{6}{|l|}{ Lymphocyte } \\
\hline Background & $38.00(1-154)$ & $7.50(1-41)$ & $14.00(5-22)$ & $.524^{\mathrm{a}}$ & $.206^{a}$ \\
\hline $\operatorname{Low}(\leq 30)$ & $1(25)$ & $5(83)$ & $5(100)$ & $.190^{\mathrm{b}}$ & $.048^{b}$ \\
\hline High (>30) & $3(75)$ & $1(17)$ & 0 & & \\
\hline Within tumor & $11.50(0-51)$ & $1.50(0-3)$ & $0.00(0-2)$ & $.999^{a}$ & $.524^{a}$ \\
\hline $\operatorname{Low}(\leq 10)$ & $2(50)$ & $6(100)$ & $5(100)$ & $.133^{b}$ & $.167^{b}$ \\
\hline High (>10) & $2(50)$ & 0 & 0 & & \\
\hline Histiocyte & $5.00(1-9)$ & $1.00(0-2)$ & $1.00(1-29)$ & $.999^{a}$ & $.999^{a}$ \\
\hline $\operatorname{Low}(<1)$ & $1(25)$ & $2(33)$ & 0 & $.999^{b}$ & $.444^{b}$ \\
\hline High $(\geq 1)$ & $3(75)$ & $4(67)$ & $5(100)$ & & \\
\hline \multicolumn{6}{|l|}{ Giant cell } \\
\hline Absent & $2(50)$ & $3(50)$ & $3(60)$ & $.999^{\circ}$ & $.999^{b}$ \\
\hline Present & $2(50)$ & $3(50)$ & $2(40)$ & & \\
\hline Neutrophil & $3.50(0-6)$ & $5.00(1-22)$ & $4.00(1-26)$ & $.999^{a}$ & $.999^{\mathrm{a}}$ \\
\hline Low $(\leq 10)$ & $4(100)$ & $4(67)$ & $4(80)$ & $.467^{\mathrm{b}}$ & $.999^{b}$ \\
\hline High (>10) & 0 & $2(33)$ & $1(20)$ & & \\
\hline
\end{tabular}

Values are presented as median (range) or number (\%).

WLV-PTC, Warthin-like variant of papillary thyroid carcinoma; PTC-LT, conventional papillary thyroid carcinoma with lymphocytic thyroiditis around the tumor; PTC, conventional papillary thyroid carcinoma without lymphocytic thyroiditis around the tumor.

${ }^{\mathrm{a} M a n n-W h i t n e y} \mathrm{U}$ test; ${ }^{\mathrm{b}}$ Fisher exact test. 
Table 3. Comparison of inflammatory cell components among the papillary thyroid cancer groups in liquid-based cytology (ThinPrep)

\begin{tabular}{|c|c|c|c|c|c|}
\hline & WLV-PTC $(n=4)$ & PTC-LT $(n=6)$ & PTC $(n=5)$ & $\begin{array}{l}\text { WLV-PTC vs PTC-LT } \\
\text { p-value }\end{array}$ & $\begin{array}{c}\text { WLV-PTC vs PTC } \\
\text { p-value }\end{array}$ \\
\hline \multicolumn{6}{|l|}{ Lymphocyte } \\
\hline Background & $38.00(17-47)$ & $23.50(6-71)$ & $4.00(2-8)$ & $.999^{a}$ & $.008^{\mathrm{a}}$ \\
\hline Low $(\leq 10)$ & 0 & $3(50)$ & $5(100)$ & $.200^{\mathrm{b}}$ & $.008^{b}$ \\
\hline High (>10) & $4(100)$ & $3(50)$ & 0 & & \\
\hline Within tumor & $14.50(5-22)$ & $3.50(0-10)$ & $0(0-0)$ & $.190^{a}$ & $.008^{\mathrm{a}}$ \\
\hline $\operatorname{Low}(<1)$ & $0(0)$ & $2(33)$ & $5(100)$ & $.467^{b}$ & $.008^{b}$ \\
\hline High ( $\geq 1)$ & $4(100)$ & $4(66)$ & 0 & & \\
\hline Histiocyte & $11.00(8-12)$ & $1.50(0-13)$ & $1.00(0-91)$ & $.048^{a}$ & $.206^{a}$ \\
\hline Low $(\leq 10)$ & $1(25)$ & $5(83)$ & $4(80)$ & $.190^{b}$ & $.206^{b}$ \\
\hline High (>10) & $3(75)$ & $1(17)$ & $1(20)$ & & \\
\hline Giant cell & & & & $.400^{b}$ & $.524^{b}$ \\
\hline Absent & $1(25)$ & 0 & $3(60)$ & & \\
\hline Present & $3(75)$ & $6(100)$ & $2(40)$ & & \\
\hline Neutrophil & $3.00(1-12)$ & $16.00(1-47)$ & $2.00(0-10)$ & $.524^{\mathrm{a}}$ & $.999^{a}$ \\
\hline $\operatorname{Low}(\leq 10)$ & $3(75)$ & $3(50)$ & $5(100)$ & $.571^{\mathrm{b}}$ & $.444^{b}$ \\
\hline High (>10) & $1(25)$ & $3(50)$ & 0 & & \\
\hline
\end{tabular}

Values are presented as median (range) or number (\%).

WLV-PTC, Warthin-like variant of papillary thyroid carcinoma; PTC-LT, conventional papillary thyroid carcinoma with lymphocytic thyroiditis around the tumor; PTC, conventional papillary thyroid carcinoma without lymphocytic thyroiditis around the tumor.

${ }^{a}$ Mann-Whitney U test; ${ }^{b}$ Fisher exact test.

\section{Statistical analysis}

The median number of inflammatory cells per one HPF was compared using the Mann-Whitney U test. Binary group distribution regarding inflammatory cell infiltration was compared using the Fisher's exact test. All statistical analyses were performed using SPSS ver. 23.0 (IBM Corp, Armonk, NY, USA) and p-values less than 0.05 were considered statistically significant.

\section{RESULTS}

The baseline clinicopathologic characteristics of each case such as age, gender, preoperative sonographic finding, preoperative FNA diagnosis, surgical pathologic diagnosis, tumor size, and associated thyroiditis are shown in Table 1. The WLV-PTC, PTC-LT, and PTC microscopic findings are shown in Fig. 1. Conventional smear and ThinPrep WLV-PTC samples showed irregular and papillary clusters with oncocytic cytoplasm and many lymphocytes within the tumor clusters and background (Fig. 1B-E) contrary to those of PTC-LT (Fig. 1G-J) and PTC (Fig. 1L-O) showing rare intratumoral and background lymphocytes. In WLV-PTC, the lymphocytic smear pattern was not different between the conventional and ThinPrep preparations.

The median number, range, and group distributions of inflammatory cells in WLV-PTC, PTC-LT, and PTC in conventional smears and in liquid-based smears are summarized in Tables 2 and 3. In conventional smears, WLV-PTC showed a tendency for association with higher numbers of lymphocytes within tumor clusters and in the background compared with PTC-LT or PTC. WLV-PTC was significantly associated with a high number of background lymphocytes compared with PTC ( $\mathrm{p}=.048)$. In ThinPrep preparations, WLV-PTC also showed a significantly higher median number of lymphocytes within tumor clusters $(\mathrm{p}=$ .008 ), in the background ( $\mathrm{p}=.008$ ), and was significantly associated with higher lymphocytes within tumor clusters $(\mathrm{p}=.008)$ and the background $(\mathrm{p}=.008)$ compared with PTC. WLV-PTC showed a tendency for association with higher lymphocytes within tumors, in the background, and with higher histiocytes. WLV-PTC showed a significantly higher median number of histiocytes per one $\operatorname{HPF}(\mathrm{p}=.048)$.

As an incidental finding, PTC-LT showed a neutrophilic smear with a higher median number compared with WLV-PTC or PTC (Tables 2, 3, Fig. 1G-J); however, the result was not statistically significant.

\section{DISCUSSION}

WLV-PTC is a rare variant of PTC having characteristic histologic features; therefore, histologic diagnosis of this tumor is usually straightforward. However, preoperative diagnosis using conventional or liquid-based cytology techniques is complicated because several diseases resemble each other based on cytologic features. The first description of WLV-PTC cytologic features 
WLV-PTC
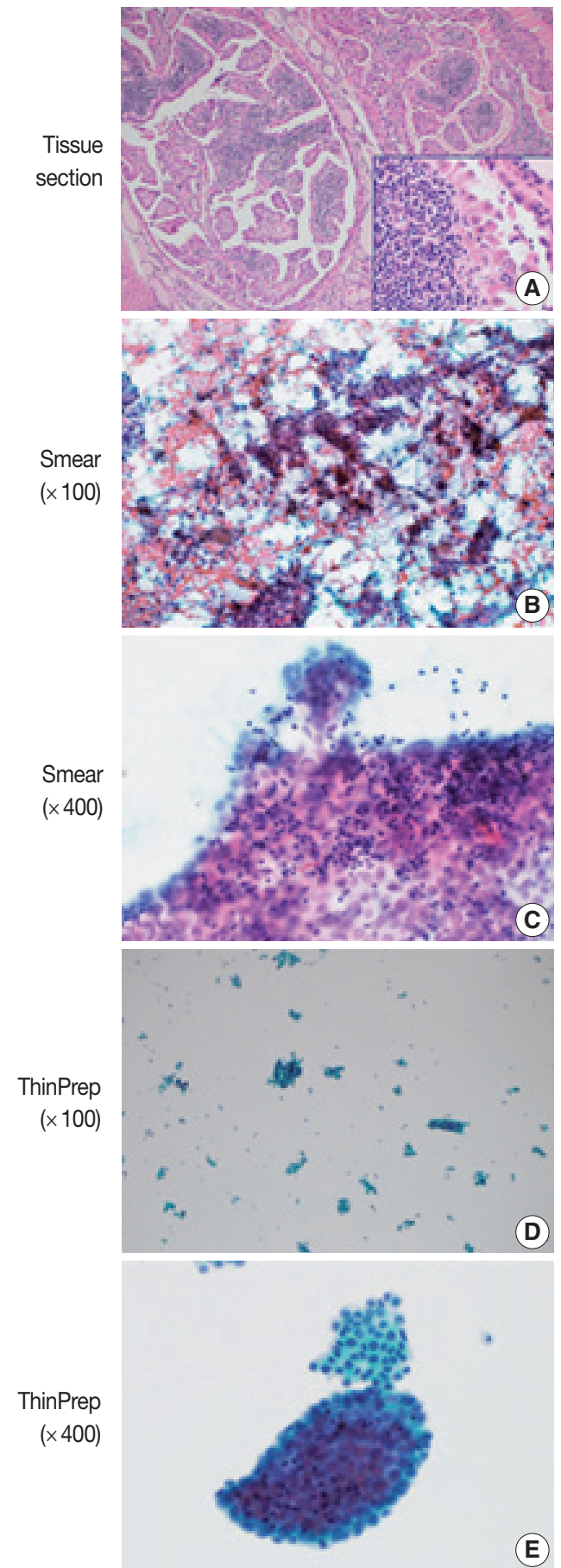

PTC-LT
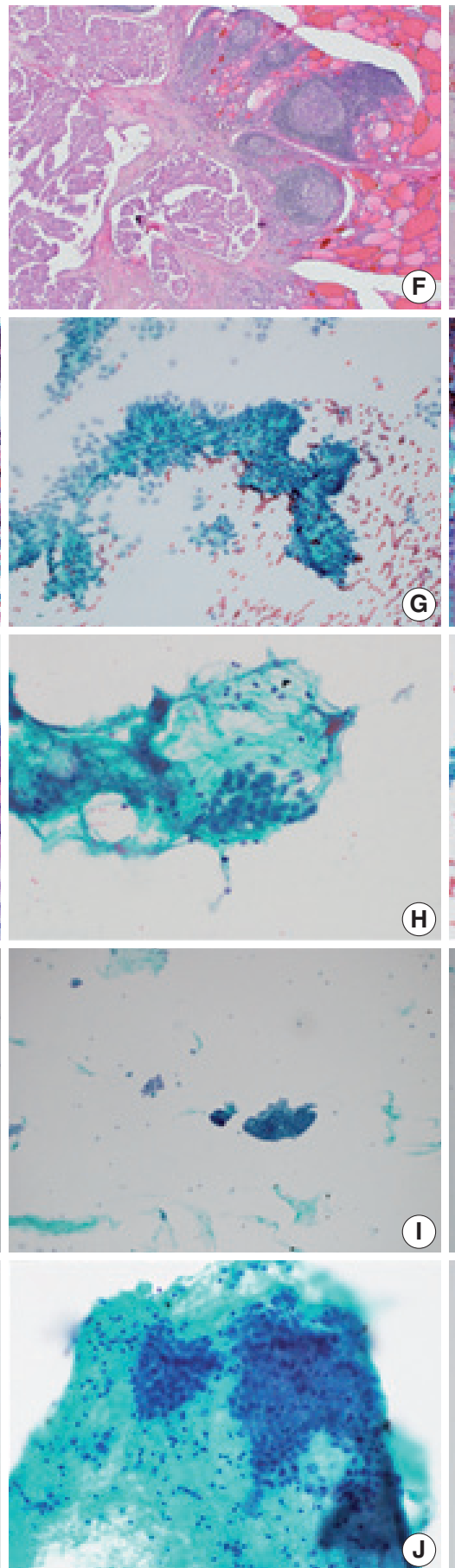

(I)
PTC
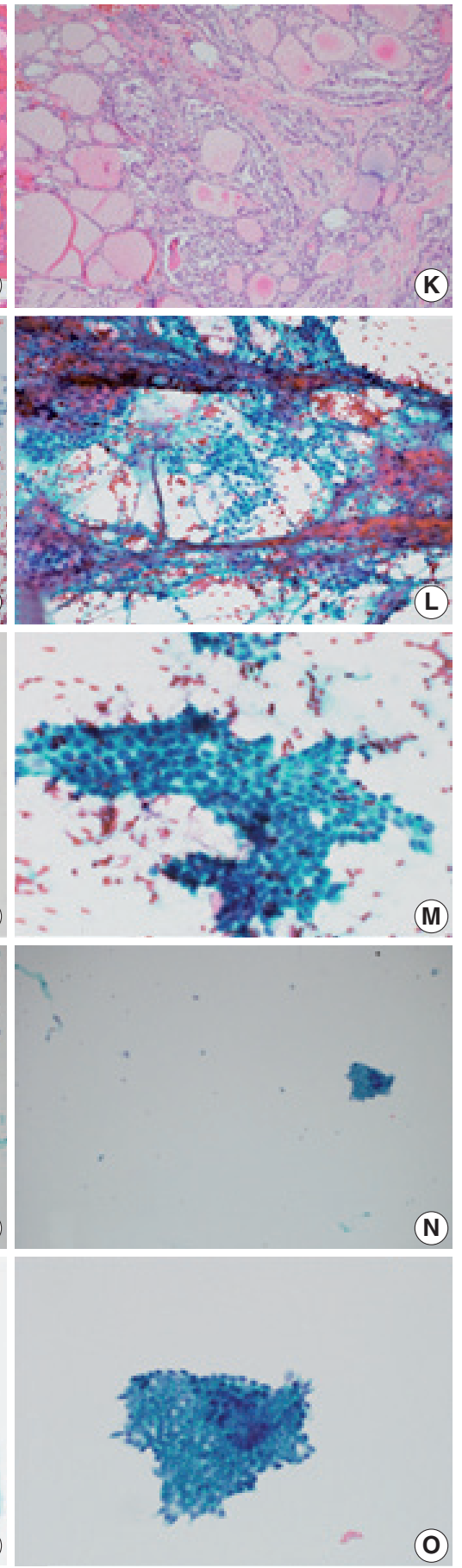

Fig. 1. Microscopic findings of Warthin-like variant of papillary thyroid carcinoma (WLV-PTC), conventional papillary thyroid carcinoma with lymphocytic thyroiditis (PTC-LT), and conventional papillary thyroid carcinoma without lymphocytic thyroiditis (PTC). (A) Histologic findings of WLV-PTC. WLV-PTC is composed of atypical follicular cells with abundant oxyphilic cytoplasm and nuclear grooves (inset) showing papillary structures with abundant lymphoid stroma. $(\mathrm{B}, \mathrm{C})$ Conventional smear of WLV-PTC showing irregular papillary clusters and numerous lymphocytes in tumor clusters in the bloody background. (D, E) Inflammatory and multinucleated giant cells are rather evenly distributed and hypercellular relative to the conventional smear. Intratumoral lymphocytes are easily seen. (F) Histologic findings of PTC-LT. (G, H) Conventional PTC-LT smear showing papillary tumor clusters without intratumoral lymphocytes, but some neutrophils seen around the tumor clusters in the lymphocytic background. (I, J) Peritumoral neutrophils seen in the lymphocytic background. (K) Histologic findings of PTC. (L-O) Hypercellular smear showing classic cytologic features of papillary carcinoma without inflammatory cells in background in both the conventional smear $(L, M)$ and in ThinPrep (N, O) preparations. 
was done by Yousef et al. in 1997. ${ }^{6}$ Since then, nuclear features typical of PTC combined with oncocytic cytoplasm and lymphocytic background were considered to be the cytologic features of WLV-PTC. Therefore, every tumor or tumor-like condition having both nuclear pseudoinclusions in follicular cells and lymphocytic background should be included on the list of differential diagnoses. This list includes Hashimoto's thyroiditis, follicular neoplasm with oncocytic change, oncocytic variant of PTC, ${ }^{7}$ PTC in the background of Hashimoto's thyroiditis, and tall cell variant of PTC. ${ }^{8}$ Paker et al. ${ }^{8}$ said the most important differential point is mixture of lymphocytes and oncocytic follicular cells, tissue fragments, and papillary structures. However, distinguishing these diseases by cytologic examination is not always clear cut. Especially in liquid-based cytology, cell clusters are easily dissolved during preparation, which can confer further diagnostic difficulty. ${ }^{?}$

In this study, we selected WLV-PTC, PTC-LT, and conventional PTC cases of which the diagnosis was confirmed by postoperative pathological examination. We compared preoperative cytology slides and investigated whether specific clues other than nuclear feature and lymphocytic background could distinguish these diseases. We focused on the component and amount of background and intratumoral inflammatory cells.

Although limitations of our study include relatively small WLV-PTC sample size due to the rarity of the disease, WLVPTC was associated with higher lymphocytes and histiocytes within tumor clusters and within the background as well. The difference was more distinct in ThinPrep preparations.

Our investigation implies that analyzing inflammatory components in preoperative cytologic diagnosis could be helpful in the differential diagnosis between WLV-PTC and conventional PTC without lymphocytic thyroiditis. If lymphocytes within tumor clusters are also considered, the preoperative diagnosis of this rare WLV-PTC variant is possible; however, further multiinstitutional studies including a large number of WLV-PTC cases will be needed.

\section{Conflicts of Interest}

No potential conflict of interest relevant to this article was reported.

\section{REFERENCES}

1. Apel RL, Asa SL, LiVolsi VA. Papillary Hurthle cell carcinoma with lymphocytic stroma: "Warthin-like tumor" of the thyroid. Am J Surg Pathol 1995; 19: 810-4.

2. Jun HH, Kim SM, Hong SW, Lee YS, Chang HS, Park CS. Warthinlike variant of papillary thyroid carcinoma: single institution experience. ANZ J Surg 2016; 86: 492-4.

3. D'Antonio A, De Chiara A, Santoro M, Chiappetta G, Losito NS. Warthin-like tumour of the thyroid gland: RET/PTC expression indicates it is a variant of papillary carcinoma. Histopathology 2000; 36: 493-8.

4. Ludvíková M, Ryška A, Korabečná M, Rydlová M, Michal M. Oncocytic papillary carcinoma with lymphoid stroma (Warthin-like tumour) of the thyroid: a distinct entity with favourable prognosis. Histopathology 2001; 39: 17-24.

5. Mai KT, Thomas J, Yazdi HM, Commons AS, Lamba M, Stinson AW. Pathologic study and clinical significance of Hürthle cell papillary thyroid carcinoma. Appl Immunohistochem Mol Morphol 2004; 12: 329-37.

6. Yousef O, Dichard A, Bocklage T. Aspiration cytology features of the warthin tumor-like variant of papillary thyroid carcinoma: a report of two cases. Acta Cytol 1997; 41(4 Suppl): 1361-8.

7. Chong Y, Suh S, Kim TJ, Lee EJ. Fine needle aspiration cytology of Warthin-like papillary thyroid carcinoma: a brief case report. Korean J Pathol 2014; 48: 170-3.

8. Paker I, Kokenek TD, Yilmazer D, Seker GE, Alper M. Oncocytic variant of papillary thyroid carcinoma with lymphocytic stroma (Warthin-like variant): report of a case with fine needle aspiration cytology and review of the literature. Cytopathology 2012; 23: 408-10. 